\title{
FEM Modelling of Ultrasound Enhanced Electrospinning (USES)
}

\section{Mäkinen, Joni}

IEEE

2019

Mäkinen , J , Helander , P , Puranen , T , Hunnakko , J , Kogermann , K , Laidmäe , I , Nieminen , H J , Salmi , A , Heinämäki , J \& Haeggström , E 2019 , FEM Modelling of Ultrasound Enhanced Electrospinning (USES) . in 2019 IEEE International Ultrasonics Symposium (IUS) . IEEE International Ultrasonics Symposium , IEEE , pp. 2511-2514 , IEEE International Ultrasonics Symposium , Glasgow , United Kingdom , 06/10/2019 . https://doi.org/10.1109/ULTSYM.20

http://hdl.handle.net/10138/326285

https://doi.org/10.1109/ULTSYM.2019.8926132

acceptedVersion

Downloaded from Helda, University of Helsinki institutional repository.

This is an electronic reprint of the original article.

This reprint may differ from the original in pagination and typographic detail.

Please cite the original version. 


\section{FEM Modelling of Ultrasound Enhanced Electrospinning (USES)}

\author{
Joni Mäkinen \\ Department of Physics \\ University of Helsinki \\ Helsinki, Finland \\ joni.mk.makinen@helsinki.fi
}

\author{
Institute of Pharmacy \\ Tartu, Estonia
}

\author{
Petteri Helander \\ Department of Physics \\ University of Helsinki \\ Helsinki, Finland
}

\author{
Ivo Laidmäe \\ Institute of Pharmacy \\ University of Tartu \\ Tartu, Estonia
}

\author{
Tuomas Puranen \\ Department of Physics \\ University of Helsinki \\ Helsinki, Finland
}

\author{
Heikki J. Nieminen \\ Department of Neuroscience and \\ Biomedical Engineering \\ Aalto University \\ Espoo, Finland
}

\author{
Jyrki Heinämäki \\ Institute of Pharmacy \\ University of Tartu \\ Tartu, Estonia
}

\author{
Edward Hæggström \\ Department of Physics \\ University of Helsinki \\ Helsinki, Finland
}

\author{
Joel Hunnakko \\ Department of Physics \\ University of Helsinki \\ Helsinki, Finland
}

\begin{abstract}
We present simulation efforts towards trying to understand ultrasound enhanced electrospinning (USES). Compared to traditional electrospinning this method uses ultrasound to produce an acoustic fountain on the free surface of the spinning liquid. This fountain is used as a base for the electrospinning process by applying a high-voltage between a collector plate and the liquid. The simulation predictions for the acoustic fountain height as a function of acoustic power are compared to measured values. Then, the effect of electrostatic deformation of the fountain, when a highvoltage is applied, is studied by simulations as a function of initial fountain height.
\end{abstract}

Keywords-ultrasound, ultrasound enhance electrospinning, electrospinning, FEM, acoustic fountain

\section{INTRODUCTION}

Electrospinning allows one to produce nano- and microscale fibers that find use in e.g. tissue engineering, wound healing, composites, filters etc. [1-3]. For such applications, ability to control the fiber diameter size is important. This can be achieved in different ways, e.g. by choosing a specific polymer solution for spinning, by changing the conductivity of the solution or the strength of the electric field, and by heating the solution etc. [1]. In previous work we demonstrated an electrospinning device/method, ultrasound enhanced electrospinning (USES), that uses an acoustic fountain instead of a needle, that is present in a traditional electrospinning set-up, as the base of a Taylor cone [4]. In the USES method the electrospinning process can be modified dynamically by varying the acoustic energy by either changing the input amplitude or the duty cycle. This enables modification of fiber diameter in a dynamic manner [4,5].
In this paper we simulated the acoustic fountain formation and the effects of the electric field on this fountain using the finite element method (FEM) with the commercial software package COMSOL Multiphysics ${ }^{\circledR}[6]$. Much work has been done on modeling the acoustic fountain formation for both reflecting and transparent fluid-fluid interfaces [7-10]. Our case is closely related to the work done on modeling sonoreactors, where the goal is to have high-intensity ultrasound form an acoustic fountain that generates atomization [10]. Modeling the electric deformation of a liquid boundary has been studied extensively and this effort has been employed to model Taylor cone and the jet formation accurately $[11,12]$.

Our work builds on these previous results. We model the USES acoustic fountain formation at the liquid-air interface, where ultrasound bursts are used instead of a continuous (standing) wave. We consider the deformation of the acoustic fountain caused by the external high voltage electric field that is essential in the electrospinning process.

\section{METHODS}

\section{A. Simulations}

Modeling our electrospinning device is a multiphysics problem. We need to consider the acoustic field, the electric field, and the surface deformation of the liquid-air interface. COMSOL provides the capability of solving the first two fields through the AC/DC and Acoustics modules, respectively. Surface deformation is considered in the CFD module in a time domain formulation. This solves for the mesh movement and surface deformation. 
Fig. 1 shows the 2D-axisymmetric electrospinner geometry used in the simulations with labels indicating the important domains and boundaries. The spinner features two chambers, chamber \#1 holds water and chamber \#2 holds the polymer solution used for electrospinning. A focusing transducer is located on boundary \#3 and the focus is approximately at the liquid-air interface, boundary \#4. Not depicted in Fig. 1 is the air domain which extends $169 \mathrm{~mm}$ above boundary \#4.

In our device we use ultrasound bursts to avoid standing waves, which is straight forward to model by solving the time dependent acoustic wave equation. However, this is time consuming and inefficient, since the time scale of the fountain formation is several orders of magnitude longer than the period of the acoustic wave. To overcome this inefficiency, we solve the acoustic field in the frequency domain. The transducer boundary condition, boundary \#3, works as a matched boundary, letting the waves that reflect from the liquid-air surface, boundary \#4, out of the domain. To solve the timeharmonic acoustic field in parallel with the time dependent Navier-Stokes equations of the CFD module, we reimplemented the Pressure Acoustics, Frequency Domain equations of COMSOL using the Weak Form PDE interface. This way the solver solves for the time-harmonic acoustic field at every time step, thus considering the correct shape of the acoustic field as the geometry deforms.

In addition to the matched boundary condition at boundary $\# 3$, an incident pressure field is applied. The amplitude of this pressure $\left(p_{0}\right)$ was determined experimentally (see appendix I) and scaled in the simulations according to the duty cycle used in experiments such that the acoustic energy is comparable:

$$
p_{0}=\sqrt{D} p_{m}\left(V_{\text {in }}\right)
$$

Here $p_{m}\left(V_{\text {in }}\right)=3.046 \mathrm{kPa} / \mathrm{V} \cdot V_{\text {in }}$ is the measured pressure and $D$ is duty cycle. The other boundary condition for acoustics is an impedance (air) boundary condition at boundary \#4. The frequency of the solved time-harmonic field was $2.7 \mathrm{MHz}$.

The electric field was modeled using the Electrostatics interface. Here the field was only considered in domain \#2 and in air. Boundary conditions were such that an electric potential

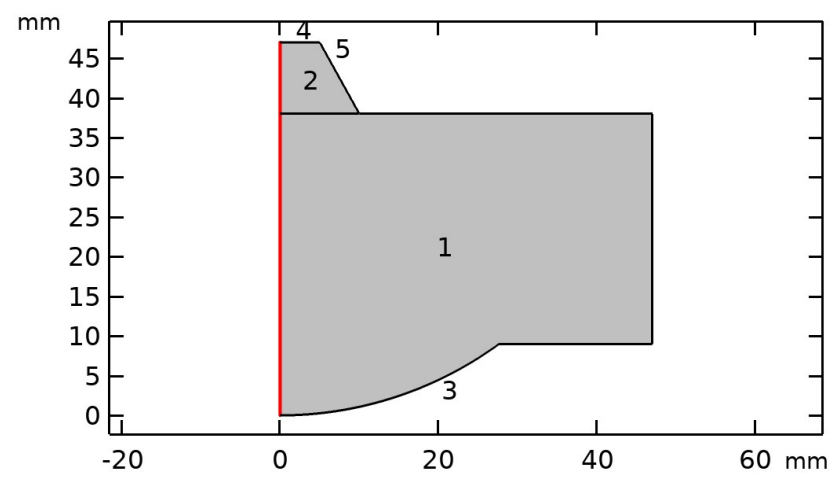

Fig. 1 2D-axisymmetric simulation geometry of the USES spinner, red line indicates the axisymmetry line. Domain $\# 1$ is water and domain $\# 2$ is the spinning liquid. Boundary \#3 is the transducer, boundary \#4 is the airliquid interface. Boundary $\# 5$ is defined as a no-slip boundary where the effect of contact angle is neglected. was applied on the bottom boundary of domain $\# 2$ and an electrical ground plane was set $169 \mathrm{~mm}$ above boundary \#4.

The Laminar Flow interface was used to compute the deformation of boundary \#4 due to acoustic radiation pressure and electrostatic pressure. These pressures on the liquid-air boundary are defined by the following equations. Acoustic radiation pressure is [9]:

$$
\begin{aligned}
& p_{R}=\vec{n} \cdot\left[\left(\left\langle T_{\text {liquid }}\right\rangle-\left\langle T_{\text {air }}\right\rangle\right) \cdot \vec{n}\right], \\
& T_{i}=-\frac{1}{2} \frac{p^{2}}{\rho_{i} c_{i}^{2}} I+\frac{1}{2} \rho_{i} \vec{u}^{2} I-\rho \vec{u}^{T},
\end{aligned}
$$

where $p_{R}$ is the acoustic radiation pressure, $\vec{n}$ is unit normal vector, $T$ is the acoustic radiation tensor, $p$ is acoustic pressure, $\rho$ is density, $c$ is speed of sound, $I$ is the identity matrix, $\vec{u}$ is the particle velocity vector and \langle\rangle denotes a time-averaged value. The electrostatic pressure is [13]:

$$
p_{E}=-\frac{1}{2} \epsilon_{0} \epsilon_{r} E_{n}^{2}
$$

where $p_{E}$ is the electrostatic pressure, $\epsilon_{0}$ is vacuum permittivity, $\epsilon_{r}$ is relative permittivity of the material, and $E_{n}$ is the normal component of the electric field evaluated on the top side of the boundary. Here the notation is such that the normal direction $\vec{n}$ of boundary \#4 is towards the positive axis direction, and the signs of the pressures (2) and (4) are such that the net force is in the normal direction. In addition to the forces (2) and (4), gravity was included in the domain as a body force, and surface tension was handled by the Laminar Flow interface.

\section{B. Measurements}

The simulation results were compared to the acoustic fountain produced by the USES spinner. The current spinner is a modified version of our previous set-up [4]. A new geometry is used (see Fig. 1) and a focusing $2.7 \mathrm{MHz}$ transducer $(\varnothing=59$ $\mathrm{mm}, 47 \mathrm{~mm}$ focal length) was used. Two cases were studied, fountain formation in (a) water and (b) in Poly(ethylene oxide) (PEO) - water solution. A 4-wt\% PEO - water solution was prepared for this. The PEO was obtained from Sigma-Aldrich and the powder in question had a molecular weight of 900.000 .

The acoustic fountains were photographed, and the resulting images were analyzed to obtain the fountain height. The analysis was done by comparing the fountains against a reference image and the height was determined by first converting the pictures to binary images with a given threshold and by then counting the height in pixels. This method produced a systematic error in the pixel counting of the size of $20 \mathrm{um}$.

\section{Material parameters}

Material parameters employed in the simulation were obtained from COMSOL's material library for water and the material parameters for the spinning liquid, PEO $4 \%$ wt, were partly obtained from measurements and partly from the literature according to Table I. The speed of sound was determined in a pulse echo experiment and density was measured to be $1000 \mathrm{~kg} / \mathrm{m}^{3}$. The literature values for PEO-water solutions were for PEO powder of molecular weight 1.000.000. 
TABLE I. MATERIAL PARAMETERS USED IN THE SIMULATIONS.

\begin{tabular}{|c|c|c|c|}
\hline & PEO solution & Water & Air \\
\hline Density $(\rho)$ & $1000 \mathrm{~kg} / \mathrm{m}^{3}$ a & $998.2 \mathrm{~kg} / \mathrm{m}^{3 \mathrm{~b}}$ & $1.2 \mathrm{~kg} / \mathrm{m}^{3}$ \\
\hline $\begin{array}{l}\text { Speed of sound } \\
(c)\end{array}$ & $1519 \mathrm{~m} / \mathrm{s}^{\mathrm{a}}$ & $1481 \mathrm{~m} / \mathrm{s}^{\mathrm{b}}$ & $343 \mathrm{~m} / \mathrm{s}$ \\
\hline Viscosity $(\mu)$ & $4.588 \mathrm{~Pa} \cdot \mathrm{s}^{[14]}$ & $1.009 \mathrm{mPa}^{*} \mathrm{~s}^{\mathrm{b}}$ & - \\
\hline $\begin{array}{l}\text { Surface tension } \\
\text { coefficient }(\gamma)\end{array}$ & $61.5 \mathrm{mN} / \mathrm{m}^{[14]}$ & $72.70 \mathrm{mN} / \mathrm{m}^{\mathrm{b}}$ & - \\
\hline $\begin{array}{c}\text { Relative } \\
\text { permittivity }\left(\epsilon_{r}\right)\end{array}$ & $82^{[15]}$ & - & 1.001 \\
\hline
\end{tabular}

\section{RESULTS}

First, acoustic fountain formation was studied in two configurations: (a) water and (b) PEO - water solution. Fig. 2 demonstrates how the acoustic field shape is captured in the simulations as the geometry deforms. Comparison between the initial and final acoustic field shape shows that the field had to be solved iteratively during the simulation. The fountain stabilization differed between the configurations due to the large change in viscosity. With water, the acoustic fountain forms quickly ( $<100 \mathrm{~ms})$ and leaves the liquid surface oscillating. On the other hand, with the highly viscous PEO-solution, the fountain formation happens slowly $(>1$ s) and reaches a maximum monotonically. The simulations were run until the water oscillations had stopped, and the fountain in PEO-solution had reached its maximum.

Figs. 3 and 4 show the results for the acoustic fountain height for the water and PEO-solution respectively as a function of input voltage to the transducer at given duty cycles. The results agreed with the measured values after an additional scaling factor was introduced. This scaling factor is explained by the burst reflecting off the bottom of the chamber and then causing an increase in effective duty cycle. The simulations don't consider absorption effects, which would account for some of the attenuation seen in the PEO-solution. By including absorption effects, one could consider heating and streaming effects that occur in the acoustic fountain. Acoustic streaming velocities are generally small [10], so the fountain shape is

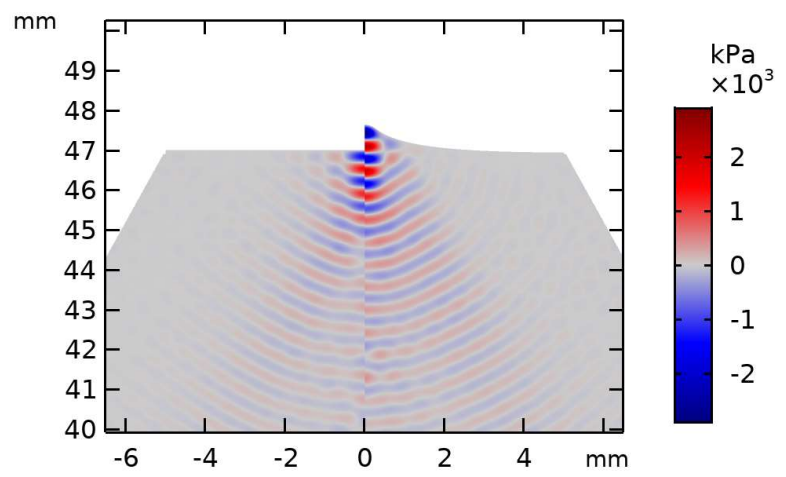

Fig. 2 Acoustic field shape before (left) and after (right) the acoustic fountain has formed and stabilized. Here the liquid is PEO-solution and the duty cycle used is $2.1 .7 \%$, corresponding to a measured fountain with a duty cycle of $7 \%$ and $\mathrm{V}_{\mathrm{in}}=19 \mathrm{~V}$.

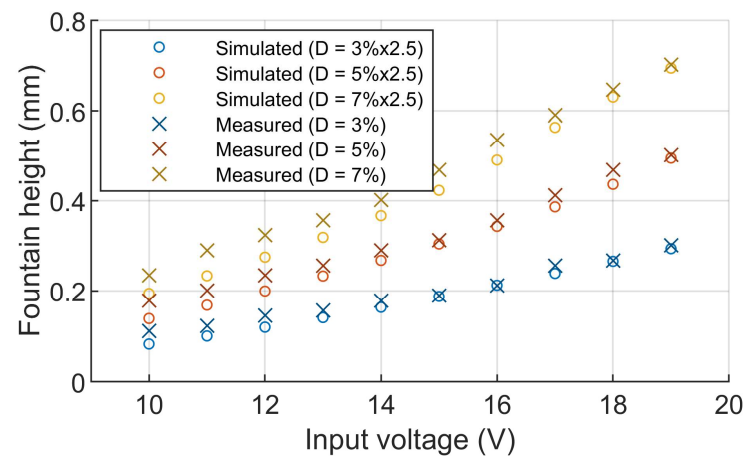

Fig. 3 Acoustic fountain height in water as a function of transducer input voltage. Three different duty cycles (D) are presented with the simulation duty cycles scaled to match the measured heights.

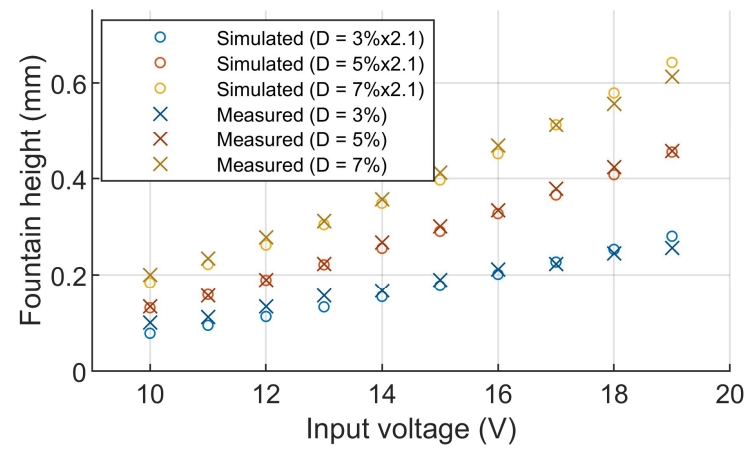

Fig. 4 Acoustic fountain height in PEO-water solution as a function of transducer input voltage. Three different duty cycles (D) are presented with the simulation duty cycles scaled to match the measured heights.

mainly determined by the radiation pressure, but temperature and flow rate do affect the diameter of electrospun fibers [1].

The fountain shape was compared to the photographed fountains. Fig. 5 shows two simulated fountain profiles overlaid on the fountain images. The shape in simulations is steeper and, in the measurements, the initial liquid surface is not flat as it was in the simulations. The fountain tip in the measurements was slightly sharper with water than with PEO solution, which agrees with the simulations.

Deformation of the acoustic fountain due to electrostatic pressure was modeled. Fig. 6 demonstrates how applying an

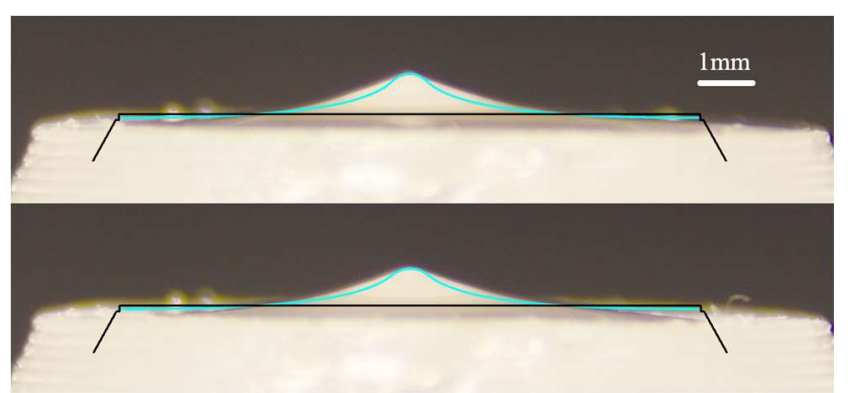

Fig. 5 Comparison of the acoustic fountain shape between simulation and measurement. (top): fountain in water. (bottom): fountain in PEOwater solution. Duty cycle is $7 \%$ in both cases, with simulation scaled according to Figs. 3 and 4 and $\mathrm{V}_{\text {in }}=19 \mathrm{~V}$. 


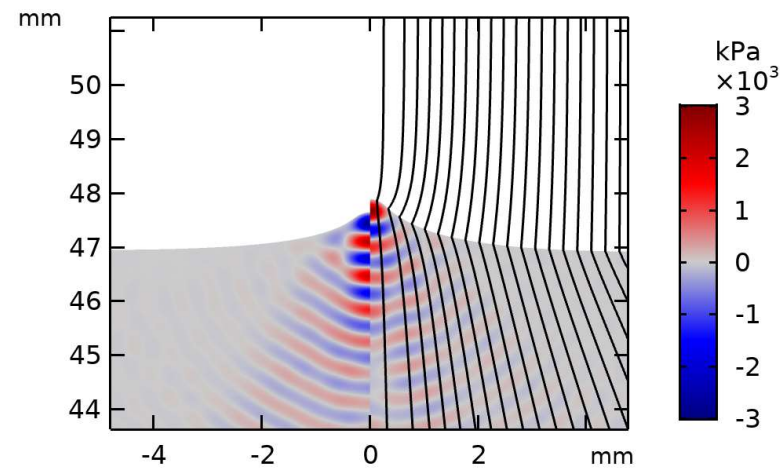

Fig. 6 Acoustic fountain from Fig. 2 (left) further deformed by the electric field (right). Electric field lines are shown.

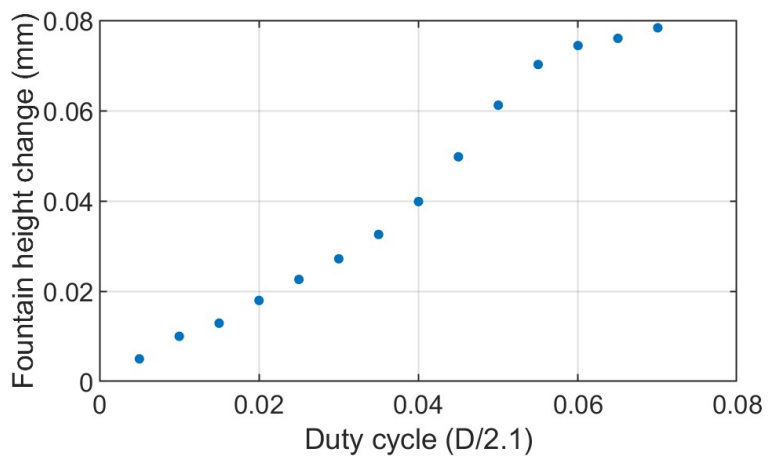

Fig. 7 Acoustic fountain height change (deformation shown in Fig. 6) in PEO solution at different duty cycles. The input voltage $V_{\text {in }}$ is $19 \mathrm{~V}$ and the high-voltage is $18 \mathrm{kV}$. As in Fig. 4, the duty cycle $(D)$ was scaled by 2.1 .

electric field deforms the acoustic fountain from its acoustic equilibrium position. Fig. 7 shows how the initial fountain height affects the magnitude of the electrostatic deformation.

\section{CONCLUSIONS}

Acoustic fountain formation in the USES set-up was modeled with FEM. Results were compared to measurements and were found to agree. Electric field induced fountain deformation was simulated at different initial acoustic fountain heights.

\section{ACKNOWLEDGMENT}

The authors thank Mr. Jere Hyvönen for his help with the hydrophone measurements of the acoustic field.

\section{APPENDIX I}

For the simulations the pressure amplitude of the focusing transducer was measured. The measurement was done using a $\varnothing$ $=0.2 \mathrm{~mm}$ needle hydrophone (Precision Acoustics NH0200). The sensitivity of the hydrophone was $38 \mathrm{mV} / \mathrm{MPa}$ at $2 \mathrm{MHz}$ and $47 \mathrm{mV} / \mathrm{MPa}$ at $3 \mathrm{MHz}$, both with $13 \%$ uncertainty. The resonance frequency of the $47 \mathrm{~mm}$ focal length transducer was $2.7 \mathrm{MHz}$, thus the sensitivity was linearly interpolated from the $2 \mathrm{MHz}$ and $3 \mathrm{MHz}$ values. The measurement was done such that an 11-cycle sine burst was sent from the transducer at different input voltages and the generated acoustic pulse was measured with the hydrophone, at $5 \mathrm{~mm}$ distance above the midpoint of the focusing transducer. From this data 4 peaks of the measured

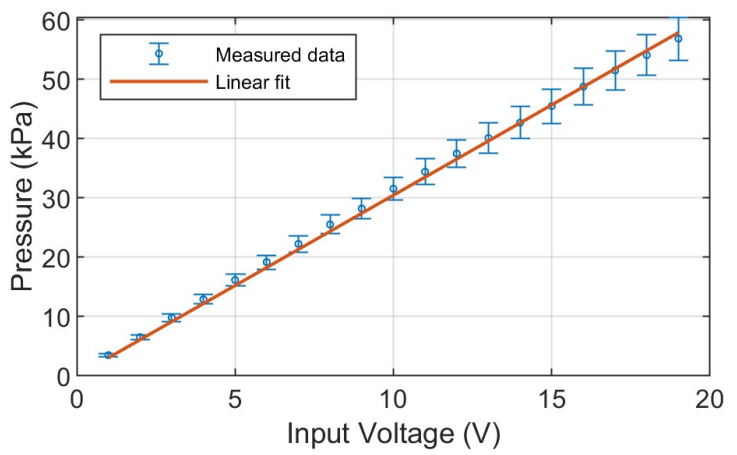

Appendix Fig. 1 Hydrophone measurement of the acoustic pressure $5 \mathrm{~mm}$ from the transducer as a function of transducer input voltage. The transducer's voltage response is linear in our region of interest.

burst were averaged at any given voltage. Appendix Fig. 1. shows the input voltage - pressure relation, where the error is defined from the $13 \%$ uncertainty and the averaging of the peaks. A linear fit through the origin was then used to determine the pressure $p_{m}\left(V_{\text {in }}\right)=3.046 \mathrm{kPa} / \mathrm{V} \cdot V_{\text {in }}$.

\section{REFERENCES}

[1] Q. P. Pham, U. Sharma and A. G. Mikos, "Electrospinning of polymeric nanofibers for tissue engineering applications: a review," Tissue Engineering, vol. 12, 2006, pp. 1197-1211

[2] K. A. Rieger, N. P. Birch and J. D. Schiffman, "Designing electrospun nanofiber mats to promote wound healing - a review," J. Mater. Chem. B, vol. 1, 2013, pp. 4531-4541

[3] Z.-M. Huang, Y.-Z. Zhang, M. Kotaki and S. Ramakrishna, "A review on polymer nanofibers by electrospinning and their applications in nanocomposites," Compos. Sci. Technol., vol. 63, 2003, pp. 2223-2253

[4] H. J. Nieminen et al., "Ultrasound-enhanced electrospinning," Sci. Rep., vol. 8,2018 , pp. $2045-2322$

[5] J. Hunnakko et al., "Towards chronic wound pads: Gradient nanofiber structure generated by ultrasound enhanced electrospinning (USES)", unpublished

[6] COMSOL Multiphysics ${ }^{\circledR}$ v. 5.4. www.comsol.com. COMSOL AB, Stockholm, Sweden

[7] J.-L. Laborde et al., "Fluid dynamics phenomena induced by power ultrasound," Ultrasonics, vol. 38, 2000, pp. 297-300

[8] B. Issenmann, A. Nicolas, R. Wunenburger, S. Manneville and J.-P. Delville, "Deformation of acoustically transparent fluid interfaces by the acoustic radiation pressure," EPL Europhys. Lett., vol. 83, 2008, sp. 34002

[9] N. Bertin, H. Chraïbi, R. Wunenburger, J.-P. Delville, and E. Brasselet, "Universal Morphologies of Fluid Interfaces Deformed by the Radiation Pressure of Acoustic or Electromagnetic Waves," Phys. Rev. Lett., vol. 109, 2012, sp. 244304

[10] Z. Xu, K. Yasuda and X. Liu, "Simulation of the formation and characteristics of ultrasonic fountain," Ultrason. Sonochem., vol. 32, 2016, pp. 241-246

[11] L. K. Lim, J. Hua, C. Wang and K. A. Smith, "Numerical simulation of cone-jet formation in electrohydrodynamic atomization," AIChE J., vol. 57, 2011, pp. 57-78

[12] M. Rahmanpour and R. Ebrahimi, "Numerical simulation of electrohydrodynamic spray with stable Taylor cone-jet," Heat Mass Transfer, vol. 52, 2016, pp. 1595-1603

[13] D. A. Saville, "Electrohydrodynamics: The Taylor-Melcher Leaky Dielectric Model," Annu. Rev. Fluid Mech., vol. 29, 1997, pp. 27-64

[14] M. W. Kim, "Surface activity and property of polyethyleneoxide (PEO) in water," Colloid Surface, Vol. 128, 1997, pp. 145-154

[15] S.A Theron, E. Zussman and A.L. Yarin, "Experimental investigation of the governing parameters in the electrospinning of polymer solutions," Polymer, Vol. 45, 2004, pp. 2017-2030

(C) 2021 IEEE. Personal use of this material is permitted. Permission from IEEE must be obtained for all other uses, in any current or future media, including reprinting/republishing this material for advertising or promotional purposes, creating new collective works, for resale or redistribution to servers or lists, or reuse of any copyrighted component of this work in other works. 